\title{
Vibration of clamped visco-elastic rectangular plate with parabolic thickness variations
}

\author{
A.K. Gupta ${ }^{\mathrm{a}, *}$ and Anupam Khanna ${ }^{\mathrm{b}}$

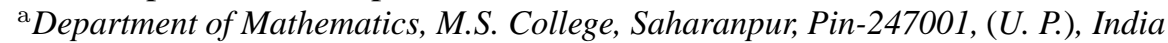 \\ ${ }^{\mathrm{b}}$ Mathematics Department, S.I.E.T. Gangoh, Saharanpur, Pin-247001, (U. P.), India
}

Received 25 September 2006

Revised 14 September 2007

\begin{abstract}
Most of the machines and engineering structures experience vibration and their design generally requires consideration for their dynamic behavior. Due to this, the study of vibration, as it deals with the vibratory behavior of bodies, is acquiring increasingly importance in several engineering applications, nuclear reactor technology and aeronautical field etc. Most of the work has been done in the field of elastic and non-elastic behavior of the bodies but a very little work is done in the field of visco-elastic bodies with varying thickness. The analysis presented here is to study the effect of taper constants on free vibration of a clamped visco-elastic rectangular plate with parabolically varying thickness. The two-dimensional thickness variation is taken as the Cartesian product of parabolic variations along the two concurrent edges of the plate. Using Rayleigh-Ritz method, frequency equation derives. Logarithmic decrement, time period and deflection for the first two modes of vibration are calculated for various values of taper constants and aspect ratio.
\end{abstract}

Keywords: Visco-elastic, rectangular plate, vibration and parabolic variation

\section{Introduction}

With the advancement of space technology, development of more and more accurate designed structure are needed and hence modern technically better designs and their analysis methods are becoming more and more critical day by day. Depending upon the requirement, durability and reliability, the materials are being developed so that they can be used to give better strength and efficiency. Since these new developed materials are frequently used in the construction of equipments and structures, therefore advancement of the application of visco-elasticity is needed to permit rational design. Applications of such materials are due to reduction of weight and size, low expenses and enhancement in effectiveness and strength.

Plates of variable thickness are often encountered in engineering applications and their use in machine design, nuclear reactor technology, naval structures and acoustical components is quite common. The consideration of visco-elastic behavior of the plate material together with the variation in thickness of the structural components not only ensure the reduction in the rate and size but also meets the desirability of high strength in various technological situations of aerospace industry, ocean engineering and electronic and optical equipments.

Several authors [1-4] have studied the effect of taper constants in two directions for elastic plate but none of them considering visco-elastic plate. Sobotka [9] has considered free vibrations of uniform visco-elastic orthotropic rectangular plates. Bhatnagar and Gupta [6,7] have studied the effect of thermal gradient on vibration of viscoelastic circular and elliptic plate of variable thickness. Ratko [10] solved the problem of transverse vibration of an eccentric circular plate. Singh and Saxena [11] have studied the transverse vibration of circular plate with

\footnotetext{
*Corresponding author. E-mail: gupta_arunnitin@yahoo.co.in.
} 
unidirectional quadratic thickness variation. Gupta and Ansari [12] have studied the transverse vibration of polar orthotropic parabollically tapered circular plate. Lal, Gupta and Reena [13] solved the problem of non-uniform orthotropic rectangular plate by quintic spline method. Lal, Gupta and Goel [14] have studied the transverse vibration of non-uniform rectangular plate by chebyshey polynomials. Ansari [15] has studied the vibration of plate of variable thickness in his thesis. Kumar [16] has studied the effect of thermal gradient on some vibration problems of orthotropic visco-elastic plates of variable thickness. Gupta and Khanna [17] solved the problem of vibration of visco-elastic rectangular plate with linearly thickness variations in both directions. Recently Gupta, Johri and Vats [18] have studied the thermal effect on vibration of non-homogenous orthotropic rectangular plate having bi-directional parabolically varying thickness. Gupta, Kumar and Gupta [19] solved the problem of vibration of visco-elastic orthotropic parallelogram plate with linearly thickness variation.

The present investigation is an another form (by considering parabolically variations) of reference [17] in which a solution was obtained in the case of linear variations of the thickness in both directions. In this paper, a mathematical model is developed for study the effect of parabolic thickness variations in both directions on the vibration of clamped visco-elastic isotropic rectangular plate. The present paper considers two-dimensional thickness variation, which is the Cartesian product of two different parabolic variations parallel to the two adjacent edges. This brings about two taper parameters. The Rayleigh-Ritz technique has been applied to determine the frequency equation by taking a two-term deflection function. It is assumed that plate is clamped on all the four edges and visco-elastic properties of the plate are of the 'Kelvin' type. The assumption of small deflection and linear, isotropic visco-elastic properties are made.

Logarithmic decrement, time period and deflection at different points for the first two modes of vibration are calculated for various values of aspect ratio and taper constants and are illustrated with graphs.

\section{Analysis and equation of motion}

The equation of motion of a visco-elastic isotropic plate of variable thickness is [17]

$$
M_{x, x x}+2 M_{x y, x y}+M_{y, y y}=\rho h w, t t
$$

with

$$
\left.\begin{array}{l}
M_{x}=-\tilde{D} D_{1}\left[w_{, x x}+\nu w_{, y y}\right] \\
M_{y}=-\tilde{D} D_{1}\left[w_{y y}+\nu w,_{x x}\right] \\
M_{x y}=-\tilde{D} D_{1}(1-\nu) w,_{x y}
\end{array}\right]
$$

Here, a comma followed by a suffix denotes partial differentiation with respect to that variable.

Using Eq. (2) in to Eq. (1) and taking the solution of equation in the form of products of two functions as, in variable separable form,

$$
w(x, y, t)=W(x, y) T(t)
$$

one obtains

$$
\begin{aligned}
& {\left[D_{1}\left(W,_{x x x x}+2 W,_{x x y y}+W,_{y y y y}\right)+2 D_{1, x}\left(W_{, x x x}+W,_{x y y}\right)+2 D_{1, y}\left(W,_{y y y}+W,_{y x x}\right)\right.} \\
& \left.+D_{1, x x}\left(W,_{x x}+\nu W,_{y y}\right)+D_{1, y y}\left(W,_{y y}+\nu W,_{x x}\right)+2(1-\nu) D_{1, x y} W,_{x y}\right] / \rho h W=-\tilde{T} / \tilde{D} T
\end{aligned}
$$

Here dot denotes differentiation with respect to t.

Equation (3) is satisfied if both of its sides are equal to a constant $\mathrm{p}^{2}$, say, and one can get two equations as,

$$
\begin{aligned}
& {\left[D_{1}\left(W,_{x x x x}+2 W,_{x x y y}+W,_{y y y y}\right)+2 D_{1, x}\left(W,_{x x x}+W,_{x y y}\right)+2 D_{1, y}\left(W,_{y y y}+W,_{y x x}\right)\right.} \\
& \left.+D_{1, x x}\left(W,_{x x}+\nu W,_{y y}\right)+D_{1, y y}\left(W,_{y y}+\nu W,_{x x}\right)+2(1-\nu) D_{1, x y} W,_{x y}\right]-\rho h p^{2} W=0
\end{aligned}
$$

which is differential equation of motion for isotropic plate and

$$
\ddot{T}+p^{2} \tilde{D T}=0
$$

is a differential equation for time-function for visco-elastic plate. 


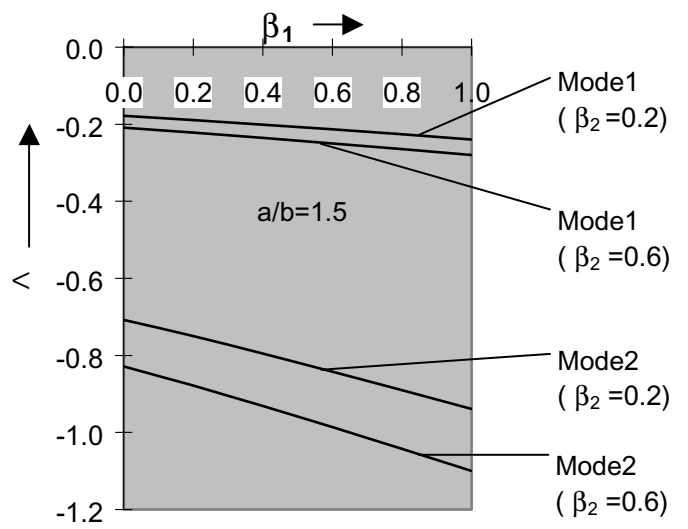

Fig. 1. Logarithmic decrement $\Lambda$ vs taper constant $\beta_{1}$ for a clamped visco-elastic rectangular plate.

\section{Solutions and deflection function}

The expressions for Kinetic energy $\mathrm{T}_{\max }$ and Strain energy $\mathrm{V}_{\max }$ are [2]

$$
T_{\max }=(1 / 2) \rho p^{2} \int_{0}^{a} \int_{0}^{b} h W^{2} d y d x
$$

and

$$
V_{\max }=(1 / 2) \int_{0}^{a} \int_{0}^{b} D_{1}\left\{(W, x x)^{2}+(W, y y)^{2}+2 \nu W,_{x x} W,_{y y}+2(1-\nu)\left(W,,_{x y}\right)^{2}\right\} d y d x
$$

Rayleigh-Ritz technique is applied to find the solution. In this technique, one requires maximum strain energy must be equal to the maximum kinetic energy. So, it is necessary for the problem under consideration that

$$
\delta\left(V_{\max }-T_{\max }\right)=0
$$

for arbitrary variations of $\mathrm{W}$ satisfying the boundary conditions which are, for rectangular plate clamped along all the four edges,

$$
\text { and } \begin{array}{r}
W=W,_{x}=0 \text { at } x=0, a \\
W=W,_{y}=0 \text { at } y=0, b
\end{array}
$$

Introducing the non-dimensional variables as

$$
X=x / a, Y=y / a
$$

In the present study it is assumed that the functional relation as represents the thickness is

$$
h=h_{0}\left(1+\beta_{1} X^{2}\right)\left(1+\beta_{2} Y^{2} a^{2} / b^{2}\right)
$$

Taking the two-term deflection function as [4]

$$
W=[X Y(a / b)(1-X)(1-Y a / b)]^{2}\left[A_{1}+A_{2} X Y(a / b)(1-X)(1-Y a / b)\right]
$$

where $A_{1}$ and $A_{2}$ are the undetermined constants associated to the shape functions.

In view of Eq. (9), Eqs (6) and (7) comes out as

$$
T_{\max }=(1 / 2) \rho p^{2} h_{0} a^{5} \int_{0}^{1} \int_{0}^{b / a}\left[\left(1+\beta_{1} X^{2}\right)\left(1+\beta_{2} Y^{2} a^{2} / b^{2}\right) W^{2}\right] d Y d X
$$

and 


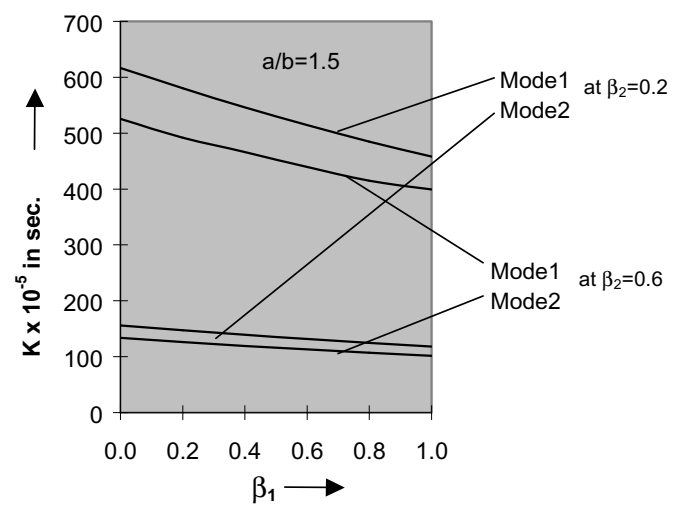

Fig. 2. Time period $\mathrm{K}$ vs taper constant $\beta_{1}$ of a clamped visco-elastic rectangular plate.

$$
\begin{aligned}
V_{\max }= & Q \int_{0}^{1} \int_{0}^{b / a}\left(1+\beta_{1} X^{2}\right)^{3}\left(1+\beta_{2} Y^{2} a^{2} / b^{2}\right)^{3}\left\{\left(\partial^{2} W / \partial X^{2}\right)^{2}+\left(\partial^{2} W / \partial Y^{2}\right)^{2}\right. \\
& \left.+2 \nu\left(\partial^{2} W / \partial X^{2}\right)\left(\partial^{2} W / \partial Y^{2}\right)+2(1-\nu)\left(\partial^{2} W / \partial X \partial Y\right)^{2}\right\} d Y d X
\end{aligned}
$$

Here limit of $\mathrm{X}$ is 0 to 1 and $\mathrm{Y}$ is 0 to b/a.

On substituting the values of $\mathrm{T}_{\max } \& \mathrm{~V}_{\max }$ from Eqs (11) and (12) in Eq. (8), one obtains

$$
\delta\left(V_{1}-\lambda^{2} p^{2} T_{1}\right)=0
$$

where

$$
\begin{aligned}
V_{1}= & \int_{0}^{1} \int_{0}^{b / a}\left[( 1 + \beta _ { 1 } X ^ { 2 } ) ^ { 3 } ( 1 + \beta _ { 2 } Y ^ { 2 } a ^ { 2 } / b ^ { 2 } ) ^ { 3 } \left\{\left(\partial^{2} W / \partial x^{2}\right)^{2}+\left(\partial^{2} W / \partial y^{2}\right)^{2}\right.\right. \\
& \left.\left.+2 \nu\left(\partial^{2} W / \partial x^{2}\right)\left(\partial^{2} W / \partial y^{2}\right)+2(1-\nu)\left(\partial^{2} W / \partial x \partial y\right)^{2}\right\}\right] d Y d X
\end{aligned}
$$

and

$$
T_{1}=\int_{0}^{1} \int_{0}^{b / a}\left[\left(1+\beta_{1} X^{2}\right)\left(1+\beta_{2} Y^{2} a^{2} / b^{2}\right) W^{2}\right] d Y d X
$$

Equation (13) involves the two unknown $\mathrm{A}_{1} \& \mathrm{~A}_{2}$ arising due to the substitution of $\mathrm{W}$ from Eq. (10). These two constants are to be determined from Eq. (13), as follows

$$
\frac{\partial}{\partial A n}\left(V_{1}-\lambda^{2} p^{2} T_{1}\right)=0, n=1,2
$$

which can simplifying as:

$$
b n_{1} A_{1}+b n_{2} A_{2}=0, n=1,2
$$

where $\mathrm{bn}_{1}, \mathrm{bn}_{2}(n=1,2)$ involve parametric constants and the frequency parameter.

For a non-trivial solution, the determinant of the coefficient of Eq. (15) must be zero. So one gets, the frequency equation as

$$
\left|\begin{array}{ll}
b_{11} & b_{12} \\
b_{21} & b_{22}
\end{array}\right|=0
$$

which is a quadratic equation in $\mathrm{p}^{2}$ from which two values of $\mathrm{p}^{2}$ can be found.

Here

$$
\begin{aligned}
b_{11}= & {\left[\left(5 \beta_{1}^{3}+132 \beta_{1}^{2}+176 \beta_{1}+154\right)\left(6 \beta_{2}^{3}+42 \beta_{2}^{2}+117 \beta_{2}+143\right) /(17342325)\right.} \\
& +(a / b)^{4}\left(6 \beta_{1}^{3}+42 \beta_{1}^{2}+117 \beta_{1}+143\right)\left(5 \beta_{2}^{3}+132 \beta_{2}^{2}+176 \beta_{2}+154\right) /(17342325) \\
& +\nu(a / b)^{2}\left(14 \beta_{1}^{3}+312 \beta_{1}^{2}+1287 \beta_{1}+1716\right)\left(14 \beta_{2}^{3}+312 \beta_{2}^{2}+1287 \beta_{2}+1716\right) /(4058104050) \\
& +(1-\nu)(a / b)^{2}\left(112 \beta_{1}^{3}+507 \beta_{1}^{2}+858 \beta_{1}+858\right)\left(112 \beta_{2}^{3}+507 \beta_{2}^{2}+858 \beta_{2}+858\right) /(1014526012.5) \\
& \left.-\lambda^{2}\left(11+3 \beta_{1}\right)\left(11+3 \beta_{2}\right) /(6930)^{2}\right]
\end{aligned}
$$




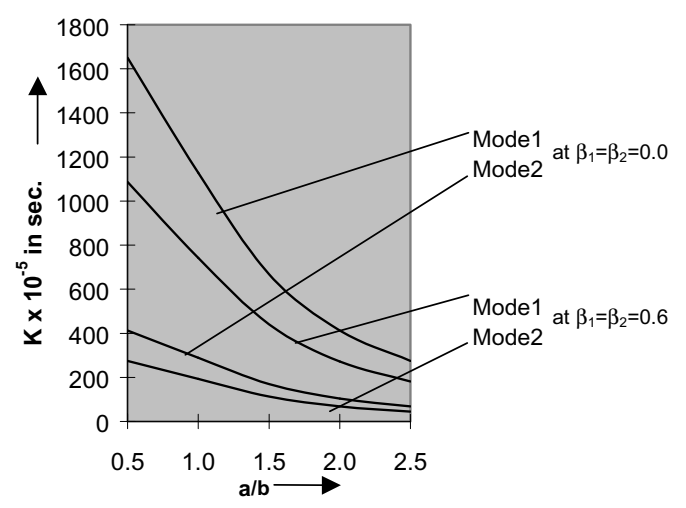

Fig. 3. Time period $\mathrm{k}$ vs aspect ratio $\mathrm{a} / \mathrm{b}$ of a clamped visco-elastic rectangular plate.

$$
\begin{aligned}
b_{12}= & b_{21}=\left[( 2 3 8 \beta _ { 1 } ^ { 3 } + 1 0 5 0 \beta _ { 1 } ^ { 2 } + 1 9 1 1 \beta _ { 1 } + 2 0 0 2 ) \left(10010 \beta_{2}^{3}+77805 \beta_{2}^{2}+235144 \beta_{2}\right.\right. \\
& +293930) /((35035)(3530687160))+(a / b)^{4}\left(10010 \beta_{1}^{3}+77805 \beta_{1}^{2}+2351441 \beta_{1}\right. \\
& +293930)\left(238 \beta_{2}^{3}+1050 \beta_{2}^{2}+1911 \beta_{2}+2002\right) /((35035)(3530687160))+\nu(a / b)^{2}\left(3 \beta_{1}^{3}\right. \\
& \left.+136 \beta_{1}^{2}+646 \beta_{1}+884\right)\left(3 \beta_{2}^{3}+136 \beta_{2}^{2}+646 \beta_{2}+884\right) /((340340)(680680)) \\
& +(1-\nu)(a / b)^{2}\left(39 \beta_{1}^{3}+204 \beta_{1}^{2}+408 \beta_{1}+442\right)\left(39 \beta_{2}^{3}+204 \beta_{2}^{2}+408 \beta_{2}\right. \\
& \left.+442) /((170170)(340340))-\lambda^{2}\left(15+4 \beta_{1}\right)\left(15+4 \beta_{2}\right) /(180180)^{2}\right], \\
b_{22}= & \left(259 \beta_{1}^{3}+1040 \beta_{1}^{2}+1716 \beta_{1}+1716\right)\left(165 \beta_{2}^{3}+1224 \beta_{2}^{2}+3570 \beta_{2}\right. \\
& +4420) /((12252240)(5005))+(a / b)^{4}\left(165 \beta_{1}^{3}+1225 \beta_{1}^{2}+3570 \beta_{1}+4420\right)\left(259 \beta_{2}^{3}\right. \\
& \left.+1040 \beta_{2}^{2}+1716 \beta_{2}+1716\right) /((12252240)(5005))+\nu(a / b)^{2}\left\{\left(6 \beta_{1}^{3}+63 \beta_{1}^{2}+221 \beta_{1}\right.\right. \\
& +286)\left(-6 \beta_{2}^{3}+21 \beta_{2}^{2}+195 \beta_{2}+286\right)+\left(-6 \beta_{1}^{3}+21 \beta_{1}^{2}+195 \beta_{1}+286\right)\left(6 \beta_{2}^{3}+63 \beta_{2}^{2}+221 \beta_{2}\right. \\
& +286) /((10010)(180180))\}+(1-\nu)(a / b)^{2}\left(30 \beta_{1}^{3}+147 \beta_{1}^{2}+273 \beta_{1}+286\right)\left(30 \beta_{2}^{3}\right. \\
& \left.\left.+147 \beta_{2}^{2}+273 \beta_{2}+286\right) /((20020)(40040))-2 \lambda^{2}\left(26+7 \beta_{1}\right)\left(26+7 \beta_{2}\right) /(72072)^{2}\right]
\end{aligned}
$$

These expressions are obtained by solving the expression for $\mathrm{V}_{1}$ and $\mathrm{T}_{1}$.

Choosing $\mathrm{A}_{1}=1$, one obtains $\mathrm{A}_{2}=-b_{11} / b_{12}$ from Eq. (15) for $n=1$, and $\mathrm{W}$ comes out as

$$
W=[X Y(a / b)(1-X)(1-Y a / b)]^{2}\left[1+\left(-b_{11} / b_{12}\right) X Y(a / b)(1-X)(1-Y a / b)\right]
$$

\section{Time functions of vibrations of visco-elastic plates}

Time functions of the plates are defined by the general ordinary differential Eq. (5) and their form depends on visco- elastic operator $\tilde{D}$.

Here authors take Kelvin's Model for which one has

$$
\tilde{D} \equiv\{1+(\eta / G)(d / d t)\}
$$

Using Eq. (18) in Eq. (5), one obtains a second order differential equation for time function T which has a solution as

$$
T(t)=e^{a_{1} t}\left[C_{1} \operatorname{Cosb}_{1} t+C_{2} \operatorname{Sinb}_{1} t\right]
$$

where $\mathrm{a}_{1}=-p^{2} \eta / 2 G$

\& 


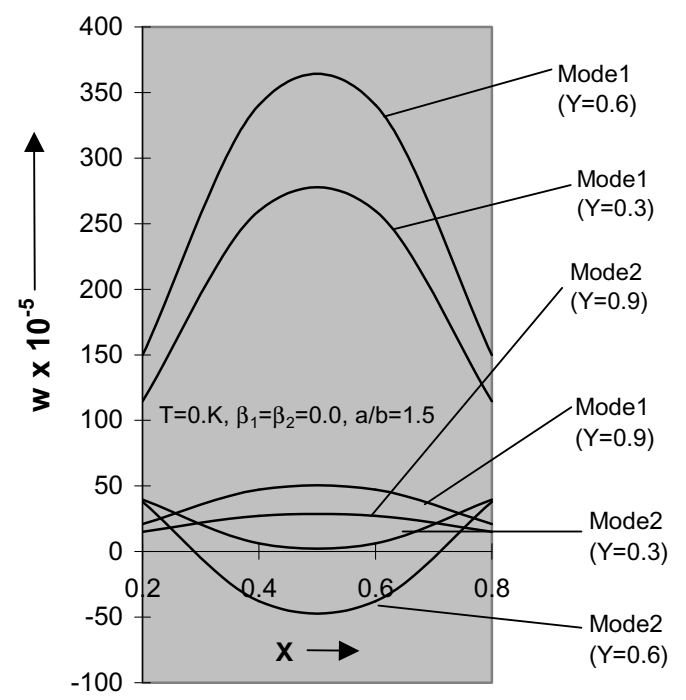

Fig. 4. Deflection w vs $\mathrm{X}$ of a clamped visco-elastic rectangular plate.

$$
b_{1}=p \sqrt{\left\{1-(p \eta / 2 G)^{2}\right\}}
$$

and $\mathrm{C}_{1}, \mathrm{C}_{2}$ are constants which can be determined easily from initial conditions which are as

$$
T=1 \& \dot{T}=0 \text { at } t=0
$$

Using Eq. (20) in Eq. (10), one obtains

$$
C_{1}=1 \& C_{2}=p^{2}(\eta / G) / 2 b_{1}=-a_{1} / b_{1}
$$

So, time function Eq. (19) comes out as

$$
T(t)=e^{a_{1} t}\left[\operatorname{Cosb}_{1} t+\left(-a_{1} / b_{1}\right) \operatorname{Sinb}_{1} t\right]
$$

Thus, deflection w may be expressed, by using Eqs (17) and (21),

$$
\begin{aligned}
w= & {[X Y(a / b)(1-X)(1-Y a / b)]^{2}\left[1+\left(-b_{11} / b_{12}\right) X Y(a / b)(1-X)(1-Y a / b)\right] } \\
& \times\left[e^{a_{1} t}\left\{\operatorname{Cosb}_{1} t+\left(-a_{1} / b_{1}\right) \operatorname{Sinb}_{1} t\right\}\right]
\end{aligned}
$$

Also time period of the vibration of the plate is given by

$$
K=2 \pi / p
$$

where $p$ is given by Eq. (16) and logarithmic decrement of the vibrations given by the standard formula

$$
\wedge=\log _{e}\left(w_{2} / w_{1}\right)
$$

where $\mathrm{w}_{1}$ is the deflection at any point on the plate at time period $K=\mathrm{K}_{1}$ and $\mathrm{w}_{2}$ is the deflection at same point at the time period succeeding $\mathrm{K}_{1}$.

\section{Results and discussion}

Numerical results for a clamped visco-elastic isotropic rectangular plate with parabolically varying thickness in both directions have been computed with accuracy by using latest computer technology. Computations have been made for calculating logarithmic decrement $\wedge$, time period $\mathrm{K}$ and deflection $\mathrm{w}$ for different values of taper constants $\beta_{1} \& \beta_{2}$ and aspect ratio $\mathrm{a} / \mathrm{b}$ for first two modes of vibration. All these results are presented in fig. 1 to fig.7.

In calculations, the following material parameters are used: 


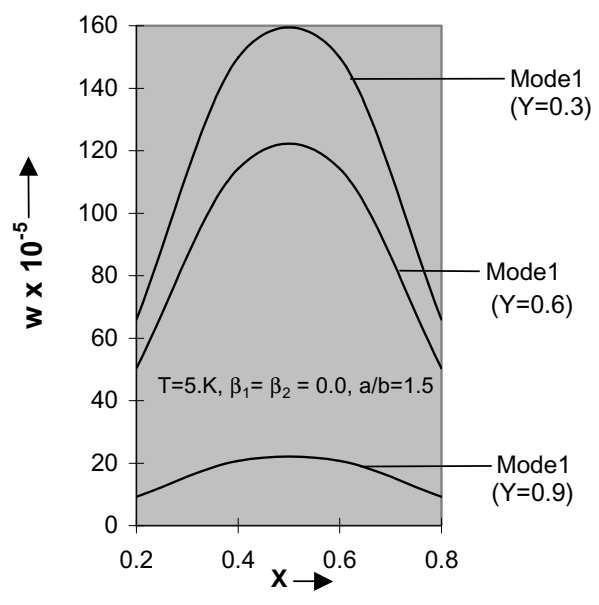

(a)

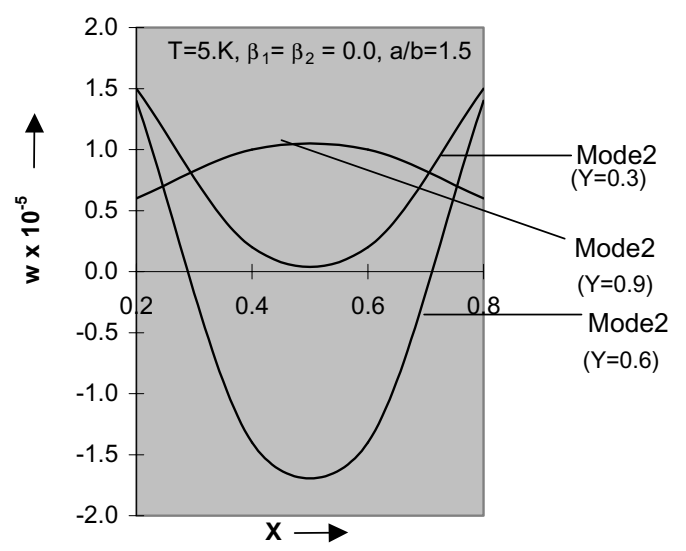

(b)

Fig. 5. (a) Deflection w vs X of a clamped visco-elastic rectangular plate (b) Deflection w vs X of a clamped visco-elastic rectangular plate.

$$
\begin{aligned}
E & =7.08 \times 10^{10} \mathrm{~N} / \mathrm{M}^{2} \\
G & =2.632 \times 10^{10} \mathrm{~N} / \mathrm{M}^{2} \\
\eta & =14.612 \times 10^{5} \mathrm{~N} . \mathrm{S} / \mathrm{M}^{2} \\
\rho & =2.80 \times 10^{3} \mathrm{Kg} / \mathrm{M}^{3} \\
\nu & =0.345
\end{aligned}
$$

These values have been reported [5] for 'Duralium'. The thickness of the plate at the center is taken as $\mathrm{h}_{\circ}=0.01$ meter

From Fig. 1, it can be easily seen that for a fixed value of aspect ratio a/b $(=1.5)$ as taper constants $\beta_{1}$ increases, logarithmic decrement $\wedge$ decreases continuously for both the modes of vibration for different values of $\beta_{2}$. It is also clear from figure that as $\beta_{2}$ increases; logarithmic decrement $\wedge$ decreases for both the modes of vibration.

Figure 2 shows a steady decrease in time period $\mathrm{K}$ with increase of taper constants $\beta_{1} \& \beta_{2}$ for fixed aspect ratio $\mathrm{a} / \mathrm{b}(=1.5)$. It is simply seen that time period $\mathrm{K}$ decreases as taper constants increase for both the modes of vibration.

Figure 3 shows time period $\mathrm{K}$ for different values of aspect ratio $\mathrm{a} / \mathrm{b}$ for both the modes of vibration for uniform thickness and non-uniform thickness for the following two cases:

$$
\beta_{1}=\beta_{2}=0.0 \& \beta_{1}=\beta_{2}=0.6
$$




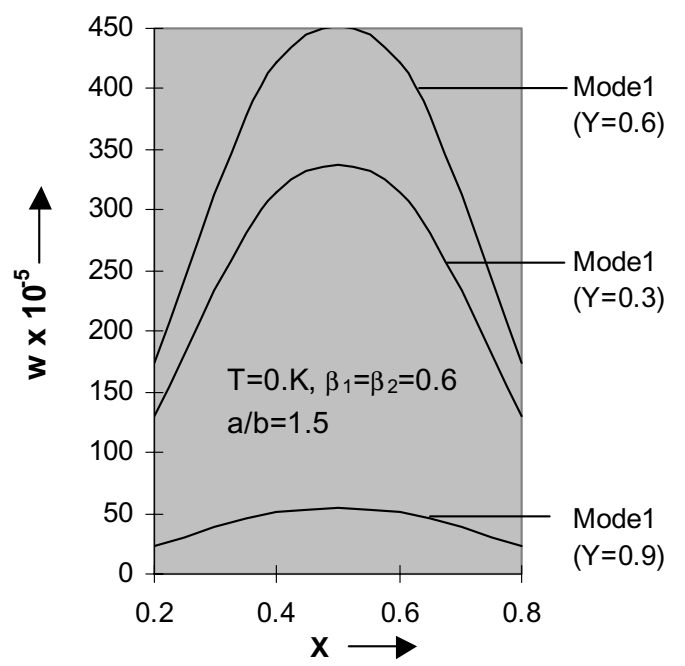

(a)

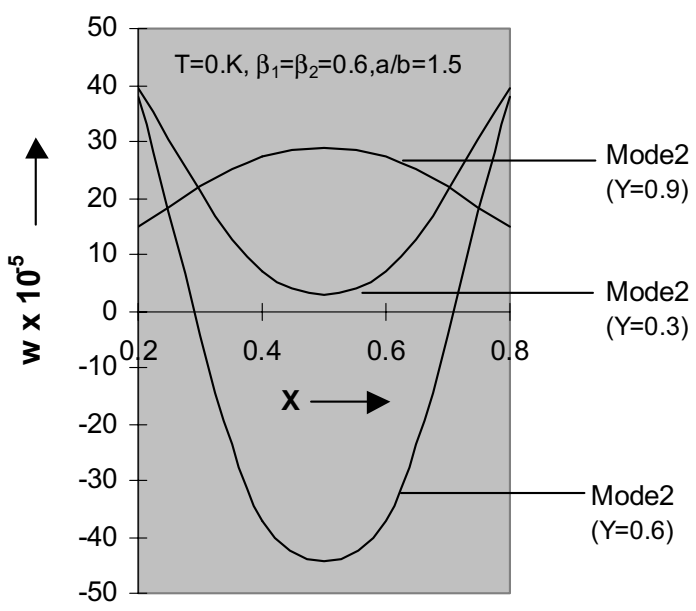

(b)

Fig. 6. (a) Deflection w vs X of a clamped visco-elastic rectangular plate (b) Deflection w vs X of a clamped visco-elastic rectangular plate.

In both the cases, one can note that time period $\mathrm{K}$ decreases as aspect ratio $\mathrm{a} / \mathrm{b}$ increases for both the modes of vibration.

Figures 4, 5, 6 and 7 respectively show the numerical values of deflection w for fixed aspect ratio a/b $(=1.5)$ for first two modes of vibration for different values of $\mathrm{X}$ (and three values of $\mathrm{Y}$ ) for the following four cases:

1. Figure 4: $\beta_{1}=\beta_{2}=0.0$ and Time is $0 . \mathrm{K}$

2. Figures $5 \mathrm{a}$ and $5 \mathrm{~b}: \beta_{1}=\beta_{2}=0.0$ and Time is $5 . \mathrm{K}$

3. Figures $6 \mathrm{a}$ and $6 \mathrm{~b}: \beta_{1}=\beta_{2}=0.6$ and Time is $0 . \mathrm{K}$

4. Figures $7 \mathrm{a}$ and $7 \mathrm{~b}: \beta_{1}=\beta_{2}=0.6$ and Time is $5 . \mathrm{K}$

Separate figs. are given for first and second mode of vibration for cases (ii), (iii) and (iv). In all above four figs., deflection $\mathrm{w}$ for first mode of vibration first increases and then decreases as $\mathrm{X}$ increases for different values of $\mathrm{Y}$ while for the second mode, w (for $Y=0.3 \& 0.6$ ) first decreases and then increases and for $Y=0.9$, w first increases and then decreases as $\mathrm{X}$ increases.

By introducing more term to expression Eq. (10), one can get results for higher modes. 


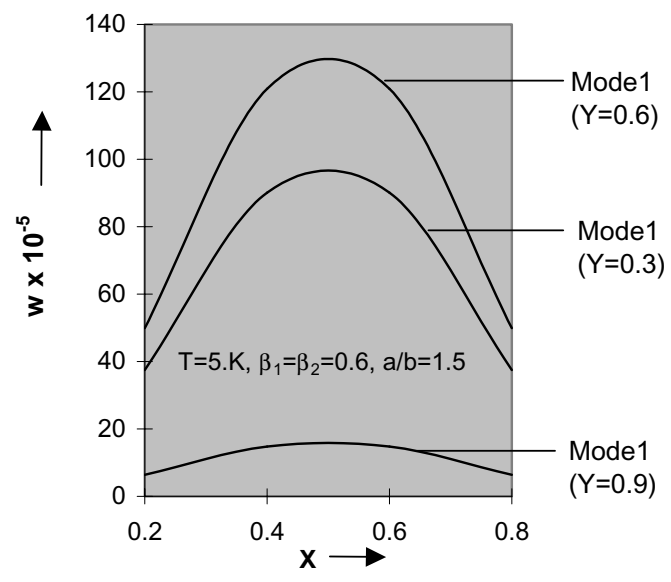

(a)

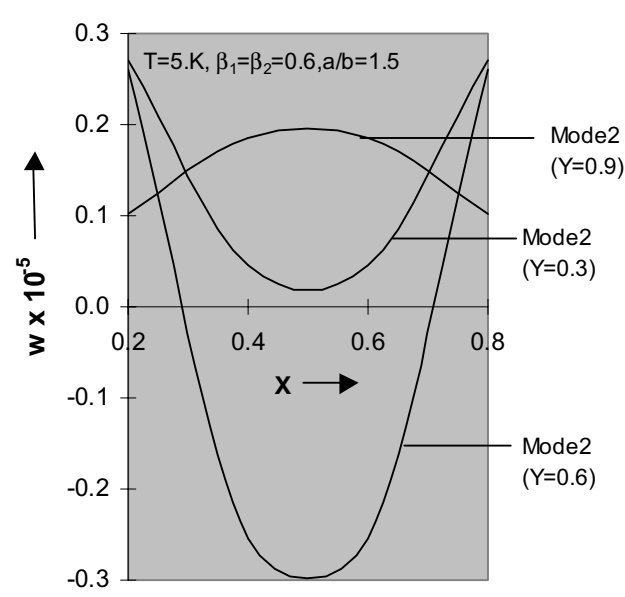

(b)

Fig. 7. (a) Deflection w vs X of a clamped visco-elastic rectangular plate (b) Deflection w vs X of a clamped visco-elastic rectangular plate.

\section{Conclusion}

The Rayleigh-Ritz technique has been applied to study the effect of the taper constants on the vibration of clamped visco-elastic isotropic rectangular plate with parabollically varying thickness on the basis of classical plate theory.

On comparison with [17], it is concluded that:

In case of plate for which thickness increases linearly, the logarithmic decrement $\wedge$ is more than that for parabolic thickness variation for both the modes of vibration for both the taper constants.

Time period $\mathrm{K}$ is more for non-uniform thickness in case of parabolic variation as comparison to linear variation.

Deflection w is less for non-uniform thickness in case of parabolic variation as comparison to linear variation.

In this way, authors concluded that parabolic variation is more useful than linear variation.

\section{Appendix: List of symbols}

a length of the rectangular plate,

b width of the rectangular plate, 


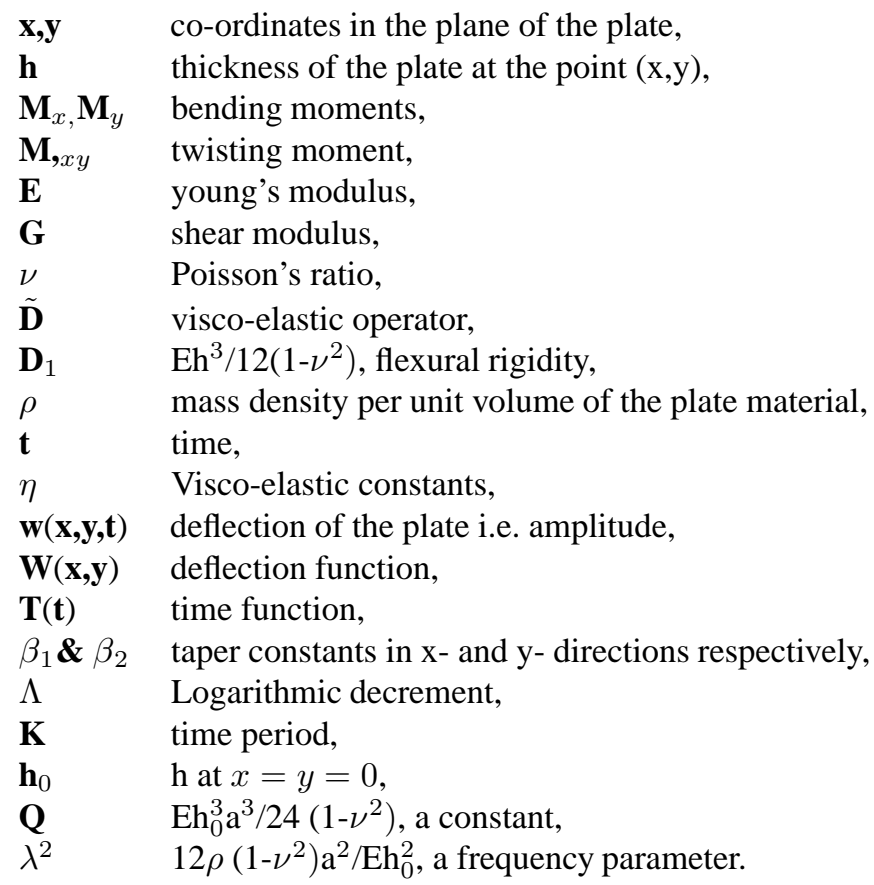

\section{References}

[1] A.W. Leissa, Recent studies in plate vibration 1981-1985 part II, complicating effects, The Shock and Vibration Dig 19(3) (1987), 10-24.

[2] A.W. Leissa, Vibrations of plates, NASA SP-160, US Govt. Printing Office, 1969.

[3] B. Singh and V. Saxena, Transverse vibration of rectangular plate with bi-directional thickness variation, J Sound and Vibration 198(1) (1996), 51-65.

[4] J.S. Tomar and A.K. Gupta, Effect of thermal gradient on frequencies of an orthotropic rectangular plate whose thickness varies in two directions, J Sound and Vibration 98(2) (1985), 257-262.

[5] K. Nagaya, Vibrations and dynamic response of visco-elastic plates on non-periodic elastic supports, J Engg For Industry 99 (1977), 404-409.

[6] N.S. Bhatanagar and A.K. Gupta, Vibration analysis of visco-elastic circular plate subjected to thermal gradient, Modelling, Simulation and Control, B, AMSE Press, 15(1) (1988), 17-31.

[7] N.S. Bhatanagar and A.K. Gupta, Thermal effect on vibration of visco-elastic elliptic plate of variable thickness, Proc. of International Conference on Modelling and Simulation, Melbourne, 1987, 424-429.

[8] P.A.A. Laura, R.O. Grossi and G.I. Carneiro, Transverse vibrations of rectangular plates with thickness varying in two directions and with edges elastically restrained against rotation, $J$ Sound and Vibration 63(4) (1979), 499-505.

[9] Z. Sobotka, Free vibration of visco-elastic orthotropic rectangular plate, Acta Technica, CSAV (6) (1978), 678-705.

[10] M. Ratko, Transverse vibration and instability of an eccentric rotating circular plate, J Sound and Vibration 280 (2005), 467-478.

[11] B. Singh and V. Saxena, Transverse vibration of a circular plate with unidirectional quadratic thickness variations, International Journal of Mechanical Science 38(4) (1996), 423-430.

[12] U.S. Gupta and A.H. Ansari, Transverse vibration of polar orthotropic parabolically tapered circular plates, Indian J Pure and App Mathematics 34(6) (2003), 819-830.

[13] R. Lal, U.S. Gupta and Reena, Quintic splines in the study of transverse vibrations of non-uniform orthotropic rectangular plates, $J$ Sound and Vibration 207(1) (1997), 1-13.

[14] R. Lal, U.S. Gupta and C. Goel, Chebyshev polynomials in the study of transverse vibrations of non-uniform rectangular orthotropic plates, The Shock and Vibration Digest 33(2) (2001), 103-112.

[15] A.H. Ansari, Vibration of Plates of Variable Thickness, PhD Thesis, University of Roorkee, 2000 (INDIA).

[16] Sanjay Kumar, Effect of thermal gradient on some vibration problems of orthotropic visco-elastic plates of variable thickness, Ph.D. Thesis, C. C. S. University, Meerut, 2003(INDIA).

[17] A.K. Gupta and A. Khanna Vibration of visco-elastic rectangular plate with linearly thickness variations in both directions, $J$ Sound and Vibration 301(3-4) )2007), 450-457.

[18] A.K. Gupta, Tripti Johri and R.P. Vats, Thermal effect on vibration of non-homogeneous orthotropic rectangular plate having bi-directional parabolically varying thickness, Accepted for Presentations in International Conference in World Congress on Engineering and Computer Science 2007 (WCECS 2007) to be held in San Francisco, USA from 24-26 October, 2007. 
[19] A.K. Gupta, Amit Kumar and D.V. Gupta, Vibration of visco-elastic orthotropic parallelogram plate with linearly thickness variation, Accepted for Presentations in International Conference in World Congress on Engineering and Computer Science 2007 (WCECS 2007) to be held in San Francisco, USA from 24-26 October, 2007. 

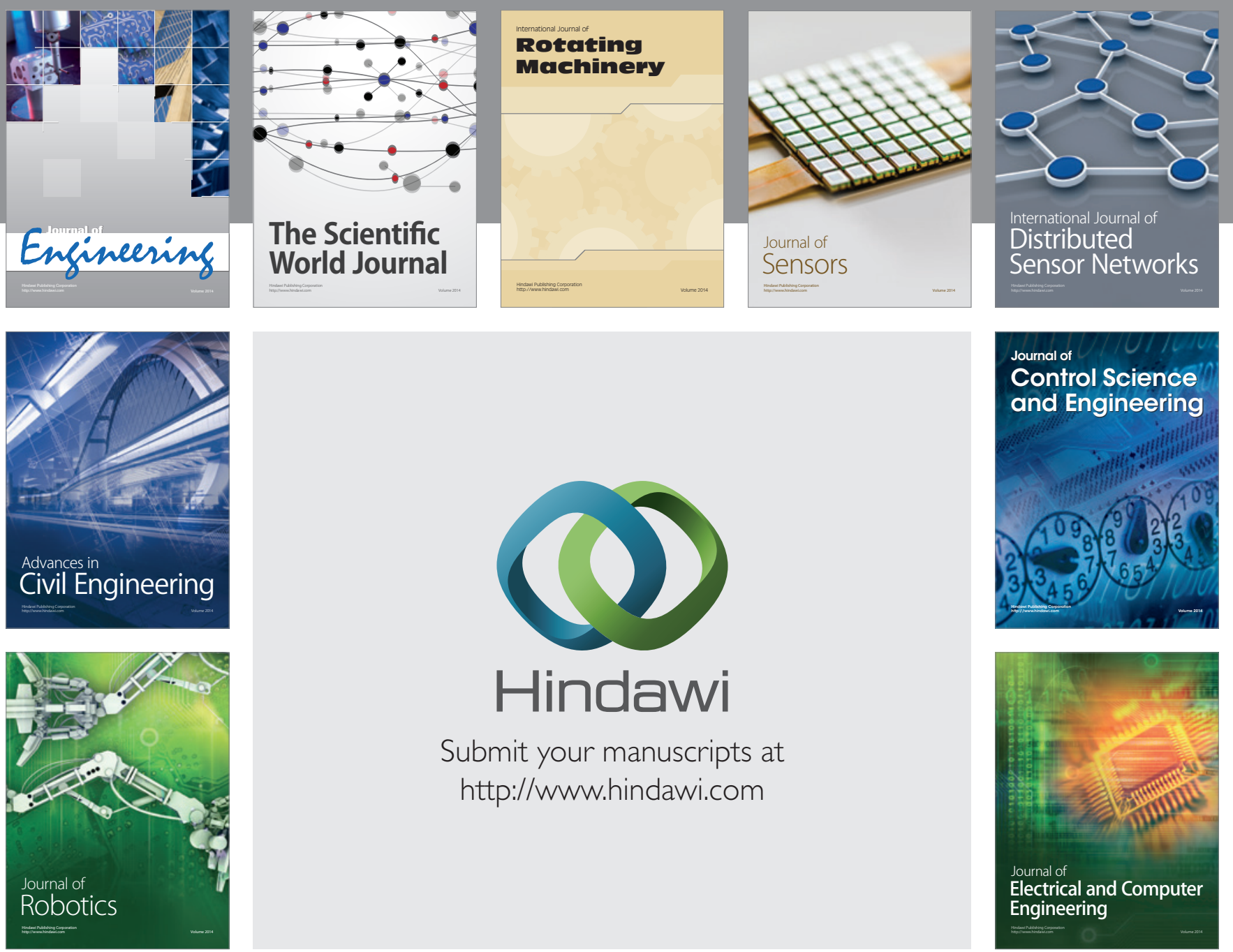

Submit your manuscripts at

http://www.hindawi.com
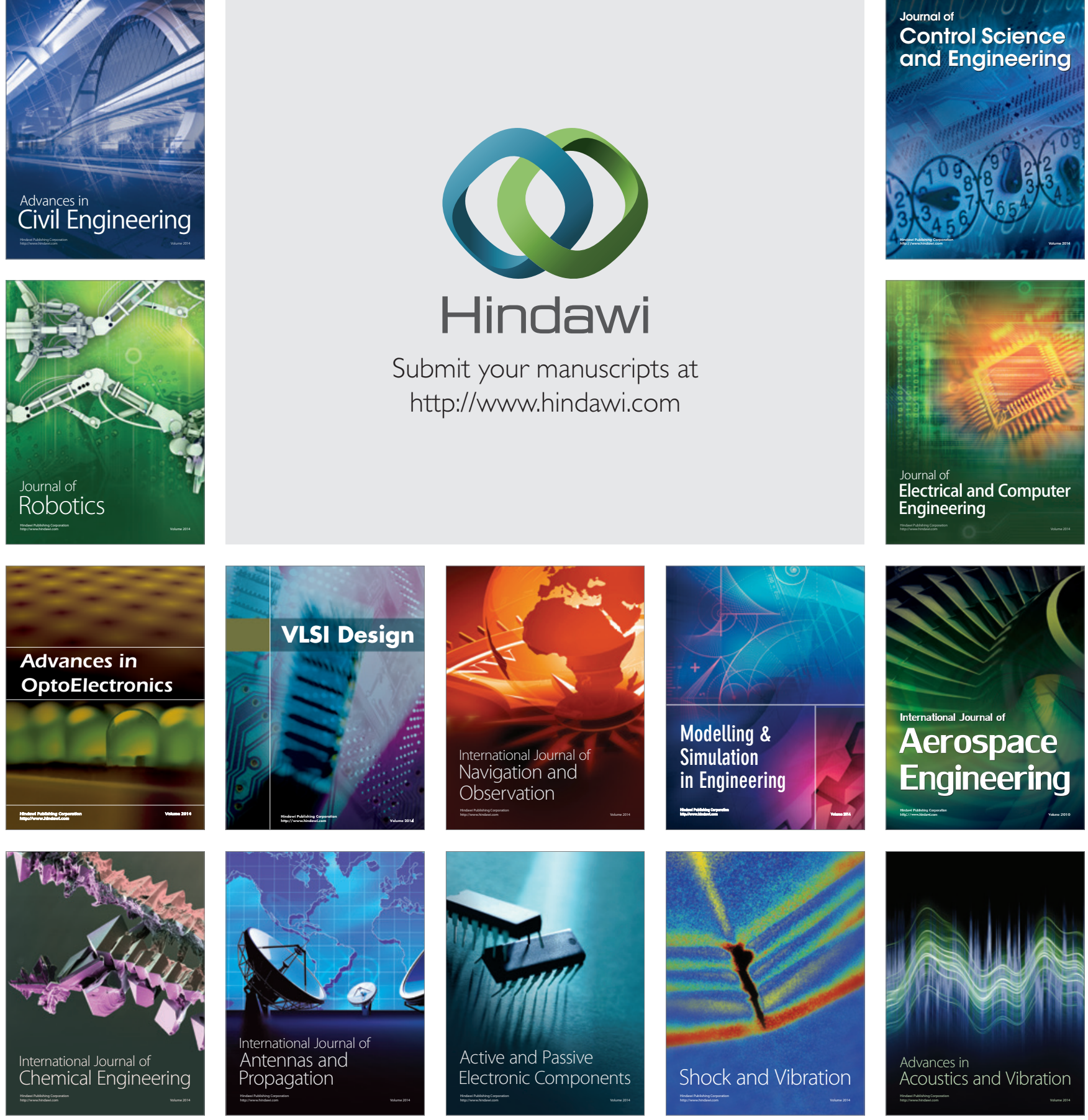